\title{
Effects of sleep loss on sustained cognitive performance during a command and control simulation
}

\author{
ROBERT G. ANGUS and RONALD J. HESLEGRAVE \\ Defence and Civil Institute of Environmental Medicine, Downsview, Ontario, Canada
}

\begin{abstract}
Because of low cognitive demands and infrequent performance assessment, previous sleep-loss studies may have provided conservative estimates of the cognitive deficits that can occur during sustained, intensive, military operations. The research described here addresses the limitations of earlier studies by requiring and measuring performance on a continuous basis in a computerized laboratory environment. During a 54-h period of wakefulness, subjects were required to continuously monitor and act upon information being transmitted over a communication network while their performance on cognitive tests, which were embedded in and distributed around the message traffic, was continuously evaluated. The results show that this cognitively demanding environment produces greater mood and performance decrements as a function of sleep loss than previous, less-demanding studies. The methodology is described in detail, and the implications of the experimental findings are discussed.
\end{abstract}

Advances in military technology (including improved night-vision devices, vehicle performance, communications systems, and firepower) and resultant changes in doctrine (emphasizing an around-the-clock capability) dictate that future conflicts will be characterized by operations of high intensity that will last for periods that exceed an individual's capability to maintain efficient performance (i.e., periods of several days to weeks). The success of such sustained operations requires: (1) that individuals perform efficiently at intense levels for extended periods, (2) that work distribution be based on individual, task, and environmental factors, (3) that performance schedules maximize the recuperative power of individuals, and (4) that commanders effectively monitor individual levels of performance in order to adjust individual capabilities to environmental demands. These requirements form the basis of a research program to study the effects of sustained operations on human performance in a military command and control environment. ${ }^{1}$

The present paper has three purposes. The first is to describe the methodology associated with a sustained operations simulation involving primarily cognitive requirements. Since both the computerized versions of the subjects' tasks and their work environment differ from previous studies, the methodology is described in detail in order to evaluate the influence of these methodological differences. The second purpose is to report some findings from an experiment that examined the degree to which mood and cognitive functioning were degraded by

Address reprint requests to either author at the Defence and Civil Institute of Environmental Medicine, 1133 Sheppard Avenue West, P.O. Box 2000, Downsview, Ontario, Canada M3M 3B9. (DCIEM No. 85-P-13)
$54 \mathrm{~h}$ of continuous, intense, cognitive demand. Although previous field and laboratory studies have shown that decrements in mood and performance occur during one or more nights without sleep, as well as over longer periods of reduced or fragmented sleep (for reviews, see Kjellberg, 1977a, 1977b, 1977c; Johnson, 1979, 1982; Johnson \& Naitoh, 1974; Naitoh, 1976; Naitoh \& Townsend, 1970; Wilkinson, 1965, 1969a, 1969b), these effects are likely conservative estimates of the sleep-deprivation-induced performance degradation that can be expected during sustained operations because of methodological difficulties. Finally, the results of the present study are contrasted with findings from previous sleep-loss studies.

The traditional approach to the study of sleep lost is to measure performance on selected tasks at various times during wakefulness. This poses several interrelated problems for studies estimating performance degradations during sustained operations conditions (cf., e.g., Ainsworth \& Bishop, 1971; Banderet, Stokes, Francesconi, Kowal, \& Naitoh, 1981; Banks, Sternberg, Farrell, Debow, \& Delhamer, 1970; Drucker, Canon, \& Ware, 1969; Haggard, 1970; Haslam, 1981, 1982; Morgan, Brown, \& Alluisi, 1974; Naitoh, 1981; Naitoh, Englund, \& Ryman, 1982; Opstad, Ekanger, Nummestrad, \& Raabe, 1978). One difficulty with such intermittent testing, which varies from measures taken every hour or two (e.g., Alluisi, 1969; Morgan et al., 1974) to only once per day (e.g. , Haslam, 1981; Opstad et al., 1978), is that relatively low frequencies of testing may not produce sufficient data to obtain reliable estimates of performance. In addition, these estimates are confounded by circadian variations in performance, with performance being best during the day and worst at night, particularly between 
0200 and 0600 h (Colquhoun, Blake, \& Edwards, 1968; Hockey \& Colquhoun, 1972).

Infrequent testing, however, may cause more serious problems. For instance, subjects may be more highly motivated to perform during infrequent, easily discriminable test periods in contrast to an environment of continuous assessment. It is also possible that subjects may be able to draw on unused reserves, or capacity, not depleted during nontest periods to enhance performance during test periods; thus, performance estimates may be spuriously high because of short-term high-energy expenditure (Harris \& O'Hanlon, 1972; Johnson, 1979; Morgan et al., 1974; Naitoh, 1976). These spuriously high performance estimates may also be augmented if the intertest intervals are relatively nondemanding, since the drain on energy reserves in general may be reduced, thereby leaving greater energy reserves for the infrequent test periods. Even if testing is continuous but low in demand, performance estimates may still be spuriously high and not generalize well to high-demand sustained operations.

A further problem with most studies is that the cognitive demand has been low because of the tasks employed. It has been observed that estimates of performance degradation are task dependent, with less cognitive and less vigilant activities reflecting less degradation as a function of sleep loss. For example, performance on simple vigilance tasks requiring sustained attention has been shown to be impaired following partial sleep deprivation (Wilkinson, Edwards, \& Haines, 1966); however, performance on simple perceptual-motor tasks has shown no visible impairment for up to $60 \mathrm{~h}$ without sleep (Wilkinson, 1964). The use of tasks that minimize cognitive demands will also lead to conservative estimates of performance degradation.

Because most of the studies noted above involved intermittent testing, nondemanding intertest intervals, and a mixture of physical and simple cognitive tasks, estimates of cognitive degradation during sustained performance conditions based on these results may be conservative. Studies providing the best estimates for a sustained operations scenario are likely those that are most continous in nature (e.g., Morgan et al., 1974; Naitoh, 1981; Naitoh et al., 1982). However, even these experiments contain long periods of inactivity, or time devoted to tasks of low cognitive demand. Probably the most demanding and most continuous work schedule was reported by Mullaney, Kripke, Fleck, and Johnson (1983). Their subjects worked continuously on a 10-min test battery of four tasks for $42 \mathrm{~h}$. Serious decrements in performance and disturbing psychological events (e.g., visual illusions, hallucinations, derealizations, and disorientations) were reported beginning approximately $18 \mathrm{~h}$ after testing began (about $2300 \mathrm{~h}$ ), which is earlier than has generally been reported in sleep-loss studies (cf. Johnson \& Naitoh, 1974). However, these results may have limited generality with respect to high-intensity sustained operations conditions because, as Mullaney et al. (1983, p. 643) pointed out, the design contained "a strong element of monotony," which is known to induce greater performance degradation (Wilkinson, 1964).

In the present study, we a dressed the limitations of previous studies by estimating the effects of sustained mental work and sleep loss on cognitive performance using a range of cognitive abilities related to command and control performance. This represents the initial stage of a research program aimed at establishing a brigadelevel command-post simulation facility capable of providing a field-exercise environment for both experimental and training purposes. Subjects worked alone in singleoperator work stations and assumed the role of operations duty officers. Their main duty was to monitor a communication network, which involved accessing, reading, understanding, interpreting, and filing information from an ongoing military conflict. In addition to monitoring and logging messages, subjects had to update tactical maps of the battle area by moving coded pins representing the different brigades' subunits. Subjects were told that the information they filed and their map updates would be used to facilitate decisions they would have to make as the scenario progressed. Embedded in and distributed around this message-processing task were a variety of cognitive tests that allowed for the continuous assessment of performance. It was intended that performance degradation would be maximized by the continuous, highdemand, mental workload and by the use of sensitive cognitive tasks. Thus, performance estimates should be less subject to the limitations of previous studies, and more pertinent for high-intensity continuous operations, especially with regard to command and control functions.

\section{METHOD}

\section{Subjects}

Twelve female students, ranging in age from 19 to 24 years (mean $=21.5$ years), were recruited from the University of Toronto. The subjects were homogeneous with respect to gender because it was intended to separate this subject pool into two groups of varying fitness levels in order to investigate the effects of physical fitness on performance degradation induced by sleep deprivation.

During the recruitment phase, subjects were given a progressive treadmill test to determine levels of physical fitness based on maximum aerobic capacity $\left(\mathrm{VO}_{2} \max \right)$, as determined by their respiratory exchange ratio, oxygen consumption, heart rate and blood lactate levels (Shephard, 1982). Six high-fit and six low-fit subjects were selected to participate in the experiment; subjects were selected as high-fit if their $\mathrm{V}_{2}$ max score was over $50 \mathrm{ml} / \mathrm{kg} / \mathrm{min}$ (mean $=53.15$ ), whereas low-fit subjects had $\dot{\mathrm{VO}}_{2 \text { max }}$ scores below $40 \mathrm{ml} / \mathrm{kg} / \mathrm{min}$ (mean $=37.83$ ). (This aspect of the study will be reported elsewhere.) All subjects received approximately $\$ 4.00 / \mathrm{h}$ for participating, were fully informed of the purpose of the experiment and procedures to be employed, and understood that they were free to withdraw from the experiment at any time. 


\section{Apparatus}

The laboratory was isolated from the normal activities of the building and contained facilities for accommodating the essential needs of both subjects and experimenters. Subjects worked individually in separate $3 \times 4 \mathrm{~m}$ experimental rooms containing a work table and chair, a wall map of the battle area, and a Digital Equipment Corporation (DEC) VT100 video display terminal. Most of the message-processing and performance tasks were generated by a DEC PDP-11/34 computer and were displayed on the subjects' video terminals; subjects responded by keying their answers into the terminals. In addition, subjects revised their strategic maps by moving coded pins, summarized the most relevant information in the messages on message cards, and filed these handwritten summaries in appropriate locations in file boxes in order to permit the later retrieval of information. Two performance tasks used auditory stimuli presented over headphones by a PDP-11/23 computer. Responses for these tasks were collected through a dedicated response panel attached to that computer. For another plotting task, spatial grid references were presented on the VT100, and subjects plotted these coordinates by placing a stylus on a second map of the battle area mounted on a Calcomp Wedge digitized tablet.

Closed-circuit television was used to visually monitor the subjects, and slave monitors displayed the information on each subject's terminal to the experimenter. The slave monitors were used because many of the subjects' responses involved only subtle hand and finger movements, which made it impossible for experimenters to ascertain by gross visual inspection whether a subject was asleep. When experimenters decided that a subject had fallen asleep, the individual was immediately awakened.

A VT100 provided experimenters with information regarding the current status of the experiment and the current position of each subject in the experimental protocol by indicating the task each subject was performing, when it started, its duration, and its serial position within the protocol. The experimenter could alter the fixed scenario in the event of equipment or other problems for any or all of the subjects by temporarily stopping the experiment, moving the subject to a different position in the protocol, restarting individual tasks or aborting the experiment. Functionally, all subjects received the same experimental protocol, but under self-paced conditions.

Continuous EEG, ECG, and core-temperature readings were recorded on two four-channel Oxford Medilog (Model 4-24) ambulatory cassette recorders. Two channels of EEG were recorded from a bipolar left- and righthemisphere parieto-occipital montage. The ECG configuration had one active electrode placed on the sternum and the other placed at about the fourth intercostal space on the subject's left side. Both EEG and ECG electrodes were referenced to the left mastoid. These data were recorded on three channels of one Medilog recorder, with the final channel used to record a timing signal and event marks that were used to facilitate synchronization of the recorded data with real-time events. Core temperature was obtained via a radio transmitter attached to a thermistor. The subject swallowed a pill containing the transmitter, and a receiver around the subject's waist picked up the signal and relayed it to the other cassette recorder.

\section{PROCEDURE}

\section{Protocol}

All subjects received the same experimental protocol in groups of three; however, they worked independently of each other and at their own pace. Subjects resided in the laboratory for 4.5 days (from Wednesday morning through Sunday afternoon). After the subjects had arrived at the laboratory, all time cues were removed from the experimenters, the environment, and the subjects, and interpersonal communication with laboratory staff was kept to a minimum. On the first day, subjects were briefed on the scenario, which included explanations of the military concepts, terminology, and procedures employed during their message-processing duties. They were also given extensive training and practice on both the messageprocessing and psychological tasks. During the afternoon, subjects were equipped for continuous, ambulatory EEG, ECG, and core-temperature recordings. Blood and urine samples were taken for biochemical analyses. (These results will be reported elsewhere.) Training continued until about $2000 \mathrm{~h}$, after which the subjects relaxed, watched a movie, and retired at about $2300 \mathrm{~h}$. They were awakened at $0700 \mathrm{~h}$ Thursday morning, began the experiment at $0900 \mathrm{~h}$ and worked continuously until $1500 \mathrm{~h}$ on Saturday (see Figure 1). In the following 24-h recovery period, subjects slept and relaxed according to their own needs.

\section{Experimental Design}

Figure 1 shows the experimental protocol. The top of Figure 1 provides a real-time description of the 54-h sleepdeprivation experiment and the preceding laboratory sleep period. As shown in Figure 1, the experiment began at $0900 \mathrm{~h}$ on Day 1 (Thursday) and ended at $1500 \mathrm{~h}$ on Day 3 (Saturday). The 54-h period of sleep deprivation consisted of nine identical 6-h performance blocks, with the same sequence of activities occurring in each block (see the bottom of Figure 1).

Each 6-h block contained four work sessions of exclusively cognitive work. These work sessions were separated by rest breaks varying from 5 to $20 \mathrm{~min}$, during which subjects were permitted to eat, drink, and use the restroom. Each session commenced with the completion of subjective self-report scales (labeled "SCALES") that examined the subjects' fatigue and sleepiness levels and mood. These scales were also presented midway through Session 1 (beginning at Hour 1) and Session 3 (beginning at Hour 4); thus, they were administered every hour of the experiment. Sessions 1 and 3 were identical and contained four message-processing trials (labeled "MES- 


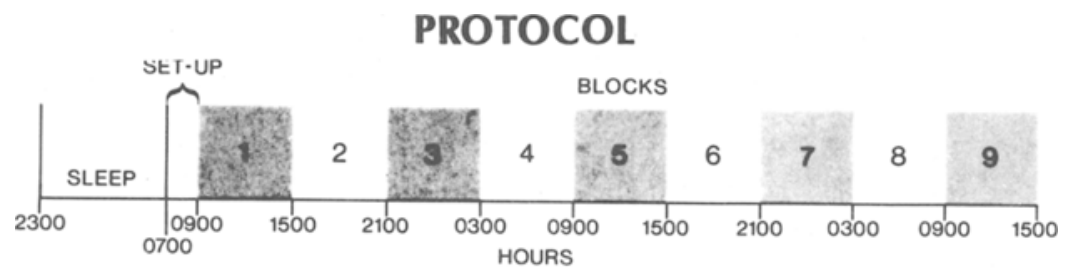

6 HOUR BLOCK

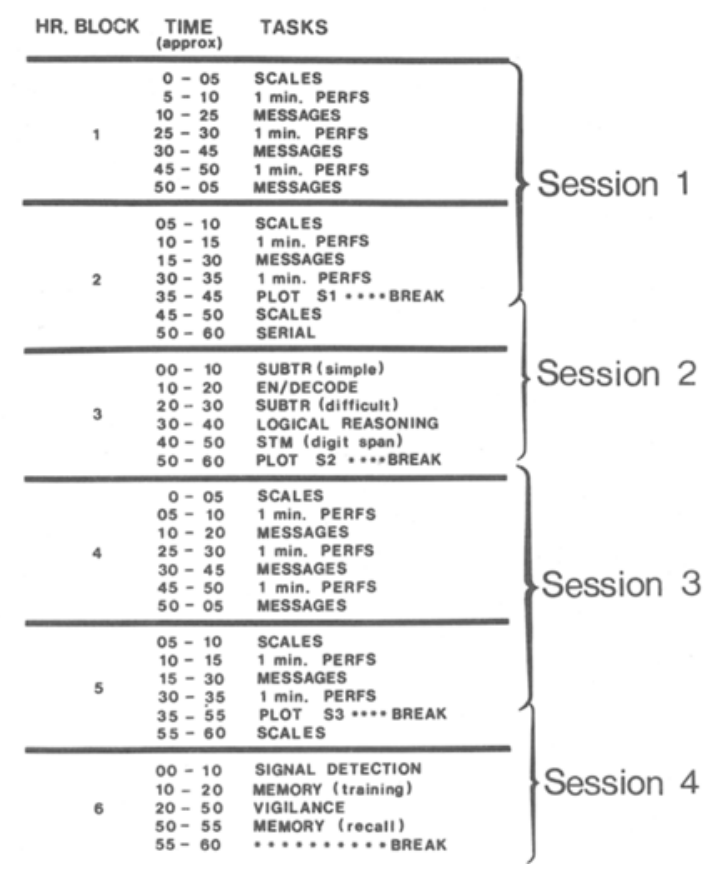

Figure 1. Experimental design. The top of the figure shows the experimental protocol, including the preexperimental night's sleep and the specific placement of the 6-h experimental blocks. The bottom of the figure shows the experimental activities and their specific temporal occurrence during each 6-h block.

SAGES') of approximately 15 min each. Immediately preceding and following each message-processing trial were five 1-min performance tasks (labeled "1-min PERFS') for a total of 10 min on each 1-min task in each 6-h block. (These tasks are described in more detail in the next section, but their order of presentation was identical to that in Session 2, i.e., serial reaction time, simple iterative subtraction, encoding/decoding, complex iterative subtraction, and logical reasoning.) These 10 dispersed 1-min trials are contrasted elsewhere with the 10 -min trials of the same tasks located in Session 2 in order to examine the influence of task duration on sleepdeprivation-induced performance decrements.

In addition to the five 10-min performance tasks, Session 2 also contained a 10-min short-term memory, digitspan task. 'Session 4 contained additional tasks, which were evaluated every $6 \mathrm{~h}$. Two of the tasks involved auditory signal detection in which the same number of sig- nals was randomly presented over either 10 min (labeled "SIGNAL DETECTION") or 30 min (labeled "VIGILANCE"). A paired-associate learning task (labeled "MEMORY training") separated the signal detection and vigilance tasks, and changes in retroactive inhibition as a function of sleep deprivation were assessed by test recall after the 30-min vigilance task. At the end of Sessions 1, 2, and 3, either Subjects 1, 2, or 3 performed a map plotting task using only a single digitized tablet (e.g., at $35 \mathrm{~min}$ into Hour 2, "PLOT S1" indicates that Subject 1 plotted on the digitizer at this time, whereas the other two subjects started their break immediately).

\section{Tasks}

For all tasks, subjects were instructed to work as quickly and accurately as possible. All responses consisted of typed keyboard responses, and the actual completed response and its duration were collected for later analysis. 
Self-report scales. Subjects completed three self-report scales each hour of the experiment (labeled "SCALES" in Figure 1). The first scale was the U.S. Air Force School of Aerospace Medicine Subjective Fatigue Checklist (Harris, Pegram, \& Hartman, 1971). To complete this scale, subjects were sequentially presented with 10 statements, such as "very lively" and "petered out," and rated themselves as "better than," "same as," or "worse than" each of these statements. Total scores on this scale covered a 20-point range, with lower scores indicating greater subjective feelings of fatigue.

The second self-report was the Stanford Sleepiness Scale (Hoddes, Zarcone, Smythe, Phillips, \& Dement, 1973). Subjects were presented with a 7-point scale, with each successive point on the scale describing an increasing level of sleepiness. Individuals selected the level of sleepiness that best described their current state, with higher scores representing greater degrees of subjectively rated sleepiness.

The third scale was the U.S. Naval Health Research Center's (NHRC) Mood Scale (Johnson \& Naitoh, 1974). To complete this scale, subjects were sequentially presented with mood-related descriptions (e.g., active, tense, considerate, happy), and they rated themselves on a 4-point scale, from "not at all" to "extremely," relative to each description. Nineteen positive and 10 negative mood descriptions were presented so that separate positive and negative mood scores could be calculated. The positive mood score covered a 57-point range; higher scores indicated a more positive mood. The negative mood score covered a 30-point range; higher scores indicated a more negative mood.

Serial reaction time (four-choice). This task (labeled "SERIAL" in Figure 1) is based on a task described by Wilkinson and Houghton (1975). Their task required subjects to cancel an illuminated light by pressing a spatially corresponding button as quickly as possible. After the light was extinguished, another light was immediately illuminated and the sequence was repeated. In the version of the task used here, there was no spatial correspondence between the stimulus and the response button. Instead, the four stimuli represented each of the four brigade HQs used in the message-processing task (i.e., "11 CMBG," "12 CMBG," "13 CMBG," "14 CMBG'). The subjects' task was to press an appropriate key, which removed the stimulus from the terminal screen and immediately replaced it with another randomly selected stimulus from the total set. The response keys were organized in a $2 \times 2$ matrix pattern (the " 4 ," " 5 ," " 1 ," and " 2 " keys on the VT100 keypad were labeled "11," " 12 ," "'13," and " 14 ," respectively). This adaptation of Wilkinson and Houghton's (1975) task enhanced the cognitive component of the task by requiring subjects to translate meaningful information into a spatially organized motor response. The duration of the trial was 10 $\min$ in Session 2 and $1 \mathrm{~min}$ in Sessions 1 and 3.

Simple iterative subtraction. This task, labeled "SUBTR simple" in Figure 1, was adapted from Hesle- grave and Furedy (1979). Subjects were presented with a randomly chosen three-digit number between 500-999 on the terminal screen followed by a randomly chosen single-digit number between 5 and 9 (e.g., "674-8"). The subjects' task was to subtract the single-digit number from the three-digit number, type in the answer, and then iteratively subtract that same subtrahend from each successively obtained difference. For the above example, the first few answers are 666, 658, and 650. To ensure that the subjects remembered each difference as well as the constant single-digit subtrahend, each three-digit answer was removed from the screen immediately after the third digit had been entered. The task lasted $60 \mathrm{sec}$ and was terminated by a "beep" from the terminal. (The warning was introduced because pilot subjects had been so engrossed in the task that they were unaware of the task's termination.) During the 10 1-min trials in Session 2 of each block, 10 different subtraction problems were presented for $60 \mathrm{sec}$ each.

Encode/decode. The encoding/decoding task (labeled "EN/DECODE' in Figure 1) is similar to that reported by Haslam $(1981,1982)$. Using a set of rules, subjects transformed six-digit grid references into four-letter codes or four-letter codes into six-digit grid references. Encoding and decoding problems were presented discretely and alternately on the screen. Each problem had to be solved using code elements printed across the top of the screen. The duration of the trial was $10 \mathrm{~min}$ in Session 2 and $1 \mathrm{~min}$ in Sessions 1 and 3.

Complex iterative subtraction. This task, adapted from Cook, Cohen, and Orne (1972) and labeled "SUBTR difficult" in Figure 1, is similar in most respects to the previously described simple iterative task except that the memory component is enhanced. Subjects were presented with only a randomly chosen three-digit number between 500-999 on the screen (e.g., 674) and received no subtrahend. Rather, their subtrahends would vary for each subtraction; the subtrahends used were taught during training. Specifically, subjects were required to subtract 9 from the presented number, then 8 from the obtained difference, 7 from the next difference, 6 from the next difference, and 5 from the next difference, and then to repeat the sequence of subtrahends for the duration of the task. (The answers for the sample problem are $665,657,650,644,639$, and then 630.) As in the simple subtraction task, the three-digit answer was removed from the screen immediately after the third digit was entered by the subject. This task forced subjects to remember each difference, as well as a varying single-digit subtrahend. Each trial lasted $60 \mathrm{sec}$ and was terminated by a "beep." During the 101 -min trials in Session 2 of each block, 10 different subtraction problems were presented for $60 \mathrm{sec}$ each.

Logical reasoning. This task (labeled "LOGICAL REASONING" in Figure 1) was devised by Baddeley (1968) and involves understanding sentences of varying syntactic complexity. In the present experiment, it consisted of individual presentations of 16 sentences (such 
as " $\mathrm{A}$ is preceded by $\mathrm{B}$ ") followed by pairs of letters (either "AB" or "BA") on each subject's terminal. The subjects were required to indicate whether or not each sentence was a true description of the pair of letters by pressing either the key marked " $T$ " ' (true), or the one marked " $F$ " (false). The 32 combinations of 16 sentences and letter pair combinations were presented in a random order without replacement until all 32 combinations had been completed. The duration of the trial was $10 \mathrm{~min}$ in Session 2 and $1 \mathrm{~min}$ in Sessions 1 and 3.

Short-term memory (digit span). During Session 2, subjects were presented with strings of digits to learn (labeled "STM digit span" in Figure 1). Each digit was presented for $1 \mathrm{sec}$, and then $0.5 \mathrm{sec}$ later the next digit was presented. The subjects' task was to memorize the complete string of digits and recall the string immediately after the last digit was presented. (The last digit was followed by a response cue.) Subjects had $3 \mathrm{sec}$ plus $3 \mathrm{sec}$ per digit to type their answer. Recall was requested in the same or the opposite order to the presentation. The trials began with a four-digit string and with the same recall order as the presentation of the four digits. If the subjects answered correctly, the string length was increased by 1 . If the answer was incorrect, the string length was decreased by 1 to a minimum of four digits. After three recall errors, the recall order reversed. The sequence continued for the duration of the 10 -min trial. The purpose of this task was to examine memory recall under both the easier forward and the more difficult backward orders.

Auditory tasks. Two auditory tasks ("SIGNAL DETECTION" and "VIGILANCE" in Figure 1) adapted from Wilkinson et al. (1966), were presented to subjects in Session 4. In the present versions, long (250-msec) and short $(200$-msec) clearly audible tones were presented with 2 -sec interstimulus intervals over headphones. The subjects' task was to detect and report the short tones, or "signals," by pressing a key on a dedicated response panel attached to the PDP-11/23 computer. Approximately 60 signals (mean $=62.63$ ) were randomly distributed over the duration of each task, with three times the signals occurring per unit time in the signal detection task. For the 10 -min signal detection task, $33 \%$ of the tones were signals; for the 30 -min vigilance task, only $11 \%$ were signals.

Paired-associate learning/recall. Prior to the vigilance assignment, a standard paired-associate learning task was presented for $10 \mathrm{~min}$ (labeled "MEMORY training" in Figure 1). In this task, subjects were presented with 12 pairs of words, which were changed in each 6-h block of the experiment. The words were all two-syllable, medium-usage, English nouns and verbs, four to eight letters in length. The words were randomly assigned to pairs. Initially, all 12 stimulus-response pairs were presented to the subjects for $4 \mathrm{sec}$ each in a fixed order. Next, the subjects were presented with each of the stimulus words in the same presentation order and attempted to respond with the appropriate response word. Subjects were given $8 \mathrm{sec}$ to respond; if no response or an incorrect response was made, the correct word was presented for $4 \mathrm{sec}$ and labeled "CORRECT RESPONSE" to provide another learning trial. If the subjects provided the correct response word for each of the stimulus words twice in the 10-min learning phase, the task was terminated; otherwise, it was terminated at the end of the 10 -min duration. Following the 30-min vigilance task, the same order of stimulus words was presented once to the subjects, and they were given $8 \mathrm{sec}$ to make each response (labeled "MEMORY recall" in Figure 1). All responses and times were collected during both the memory training and recall phases.

Map plotting. In the map plotting task, six-digit grid references were presented on the terminal, and the subjects had to plot these coordinates, using a stylus, on a map of the battle area in the scenario that was mounted on a Calcomp Wedge digitized tablet. The stimulus locations were selected randomly from the map grid area and presented individually on the terminal. Each stimulus location remained on the screen until the stylus contacted the map; a new grid reference was presented to the subject when the plotted coordinates were registered by the computer. The task lasted $5 \mathrm{~min}$, and response time and accuracy measurements were collected. Due to the availability of only a single digitizing tablet, each subject plotted only once in each 6-h block (e.g., at 35 min into Hour 2, "Plot S1" in Figure 1 indicates that Subject 1 plotted on the digitizer at this time, while Subject 2 plotted at 50 min into Hour 3, and Subject 3 plotted at 35 min into Hour 5).

Message processing. The task required subjects to monitor a communication network, which involved accessing, reading, understanding, interpreting, and filing information from the simulated military conflict. To access the message information, subjects monitored two numerical queues displayed near the top left corner of the terminal screen. These values represented the cumulative number of messages in two queues of different priority. The subjects were instructed to always access messages from the Priority 1 queue, if messages were available, regardless of the number of Priority 2 messages. A message appeared in one of the two queues about every $95 \mathrm{sec}$ for 9 min of the 15-min session, with the Priority 2 messages outnumbering the Priority 1 messages by a ratio of 8:1. Because subjects were instructed to access available Priority 1 messages immediately after completion of the current message being processed, the occurrence of these messages was relatively constant across the duration of the experiment, varying primarily with the subjects' access rate. For the messages to appear on the terminal screen, subjects pressed one of two appropriately labeled keys on their keyboard. This caused the earliest message in the queue to be presented.

Once the subjects had accessed each message, the task was to read and understand it well enough to answer a set of questions which followed the message. The Priority 1 questions required the subjects to decode resource states of various units by changing a four-letter code into a six- 
digit code using a set of rules that were similar to the rules used in the decoding portion of the encode/decode task; data from these questions will be presented in the results. Priority 2 questions required the subject to perform such duties as identifying the locations of various units (using the map grid references), describing units' activities (current or intended), selecting the most appropriate unit for a specific task, calculating equipment resources, and estimating travel distances and times of arrival. Most of the questions required short phrases to be typed on the keyboard, but some required that the tactical maps of the battle area be updated by moving coded pins representing the different brigades' subunits. (Map reading and plotting accuracy were evaluated by asking subjects to type six-digit map coordinates of specific units on the terminal at various times during the experiment.) Other questions required that summaries be written and manually filed. Accurate completion of the written summaries was important, since previously processed messages could not be retrieved from the computer and manually filed information was necessary to answer questions asked in later messages. Message-processing tasks occurred during Sessions 1 and 3 in each 6-h block.

\section{RESULTS}

In order to contrast the estimates of performance degradation obtained from this experiment with estimates reported in previous, less cognitively demanding studies that used intermittent testing, only data from comparable cognitive tasks will be reported. Specifically, data from the self-report fatigue, sleepiness, and mood scales are presented, as well as data from the 10-min versions of the serial reaction time, logical reasoning, encode/decode, and 30-min auditory vigilance tasks. In addition, some data from the message-processing task are also presented. This task marks an initial attempt to collect data from a sustained, relatively continuous, and progressive cognitive task that was presented for $1.5 \mathrm{~h}$ in every $3 \mathrm{~h}$. Included as part of these data are results from the relatively unobtrusive, "resource decoding" (Priority 1) messages embedded within the message-processing task. All scales and tasks were analyzed using analysis of variance, with the 12 subjects divided into two groups of 6 highand 6 low-fit subjects.

\section{Self-Report Scales}

The scales were administered every hour in the following order: fatigue, sleepiness, and mood. The analyses revealed significant changes in all scales over the nine experimental blocks. Figure 2 shows the data for all scales, with each data point representing the mean of the six scores obtained in each 6-h block. Larger numbers on the ordinate (Figure 2) represent less subjective fatigue, greater subjective sleepiness, greater positive subjective mood, and greater negative subjective mood.

The upper left-hand panel of the figure indicates that subjects became progressively more fatigued over the experiment, as indicated by lower scores $[\mathrm{F}(8,40)=29.08$, $\mathrm{p}<.001]$. However, the fatigue effect became acute after $18 \mathrm{~h}$ into the experiment (about $0300 \mathrm{~h}$ ) and then plateaued for about $24 \mathrm{~h}$. Following this plateau, another dramatic decline occurred from 0300 to $0900 \mathrm{~h}$ during the second night.

The other scales showed similar patterns of change with similar plateaus during the second day. The lower lefthand panel of Figure 2 shows that there were significant increases in subjective sleepiness (measured by the Stanford Sleepiness Scale) over the experiment $[F(8,40)=$ $33.91, \mathrm{p}<.001$ ], with dramatic increases in sleepiness occurring at 18 and $42 \mathrm{~h}$ into the experiment. The right side of Figure 2 shows the changes in both components of the NHRC Mood Scale. There was a significant decline in positive affect over the course of the experiment, as reflected by the lower positive scores $[F(8,40)$ $=30.32, \mathrm{p}<.001]$, and a significant increase in negative affect, as indicated by the increasing negative mood scores $[F(8,40)=35.08, p<.001]$.

Although significant changes in self-reported fatigue, sleepiness, and mood were anticipated (although not the specific topography showing second-day plateaus), the specific design of the experiment allowed estimates of these self-reported states gathered from within work sessions to be contrasted with those obtained following a rest period. This difference conceptually parallels the difference between continuous and intermittent testing; if intermittent testing produces a conservative estimate of degradation in performance and mood, as was argued in the introduction, then testing following a rest period should result in a conservative estimate of degradation relative to an estimate obtained during a work session.

Subjects completed the self-report scales every hour, that is, once at the beginning of each of the four work sessions in each 6-h block following a rest period and once during Sessions 1 and 3. To examine whether there were any differences on the self-report scales following a rest period in contrast to during a work session, the scales at the beginning and in the middle of Sessions 1 and 3 were compared.

Although all scales showed significant changes over the course of the experiment (see Figure 2), the scales also showed differential estimates of the subjects' levels of fatigue, sleepiness, and mood, depending on when these subjective feelings were assessed. If the scales were administered during a work session, subjects reported feeling worse than if the scales were administered after a short rest period, regardless of the time of day after the first $18 \mathrm{~h}$. In addition, this difference was most apparent during the first night of sleep loss and the second day. Consider the upper left-hand panel of Figure 3. In that panel, it can be seen that the subjects reported feeling significantly more fatigued during the work session than following a short rest $[\mathrm{F}(1,5)=48.94, \mathrm{p}<.001]$. This difference becomes apparent only about $18 \mathrm{~h}$ into the experiment. It becomes pronounced during the first night and is maintained to a lesser degree over the remainder 

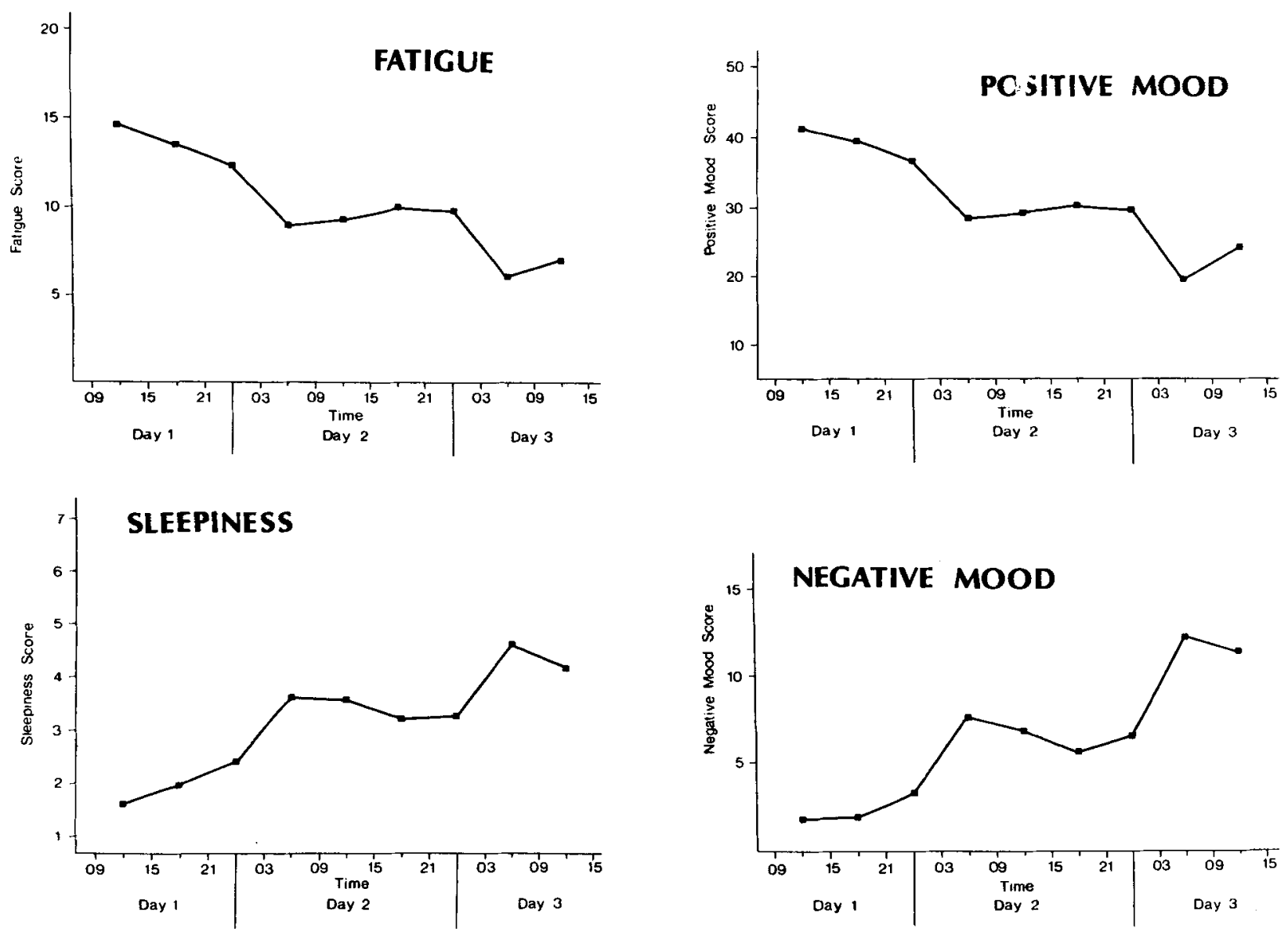

Figure 2. Self-report scales. The figure shows the changes over the nine experimental blocks in the subjective levels of fatigue, sleepiness, and positive and negative mood; each data point represents the mean of the six scores obtained in each 6-h block. Larger numbers on the ordinate represent less subjective fatigue, greater subjective sleepiness, a more positive subjective mood, and a more negative subjective mood.

of the experiment, as indicated by the significant interaction between the two curves over the 18 messageprocessing sessions of the experiment $[F(17,85)=2.78$, $\mathrm{p}<.005]$.

The lower left-hand panel of Figure 3 shows that the subjects reported significantly greater subjective sleepiness when asked during a work session $[F(1,5)=21.89$, $\mathrm{p}<.01]$, and a significant interaction between the two curves occurred over the experiment $[F(17,85)=3.15$, $\mathrm{p}<.005]$. The differences between the two sleepiness estimates were similar to the differences observed for the subjective fatigue data reported earlier. The right side of the figure shows the changes in both components of the NHRC Mood Scale. Subjects reported a significantly lower positive $\operatorname{mood}[\mathrm{F}(1,5)=11.69, \mathrm{p}<.05]$ and $\mathrm{a}$ significantly greater negative mood $[F(1,5)=22.50, p$ $<$.01] during the work sessions. These differences emerged only after $18 \mathrm{~h}$ into the experiment, as indicated by the significant interaction of both the positive $[\mathrm{F}(17,85)$ $=1.98, \mathrm{p}<.05]$ and the negative $[\mathrm{F}(17,85)=4.26$, $\mathrm{p}<.001]$ moods with time.
Once the initial decline in subjective state commenced at about $0300 \mathrm{~h}$ of the first night, the curves for the scales after a rest and during a work period began to diverge and did not overlap for the remainder of the experiment. The two curves diverged to the greatest extent during the second day (i.e., after more than $24 \mathrm{~h}$ awake). During the second night, the curves converged again; it appeared that the subjects were so fatigued that rest breaks did little to revive them.

\section{Cognitive Performance Tasks}

The data collected during the continuous 10-min serial reaction time, logical reasoning, and encoding/decoding tasks in each of the nine blocks of the experiment (Hours 2 and 3 in Figure 1) are reported along with the data from the 30-min auditory vigilance task (Hour 6 in Figure 1). These data represent the most common tasks reported in previous sleep deprivation studies. For the analysis, the continuous 10-min tasks were broken down into 101 -min periods on the basis of correct responses and errors, since 

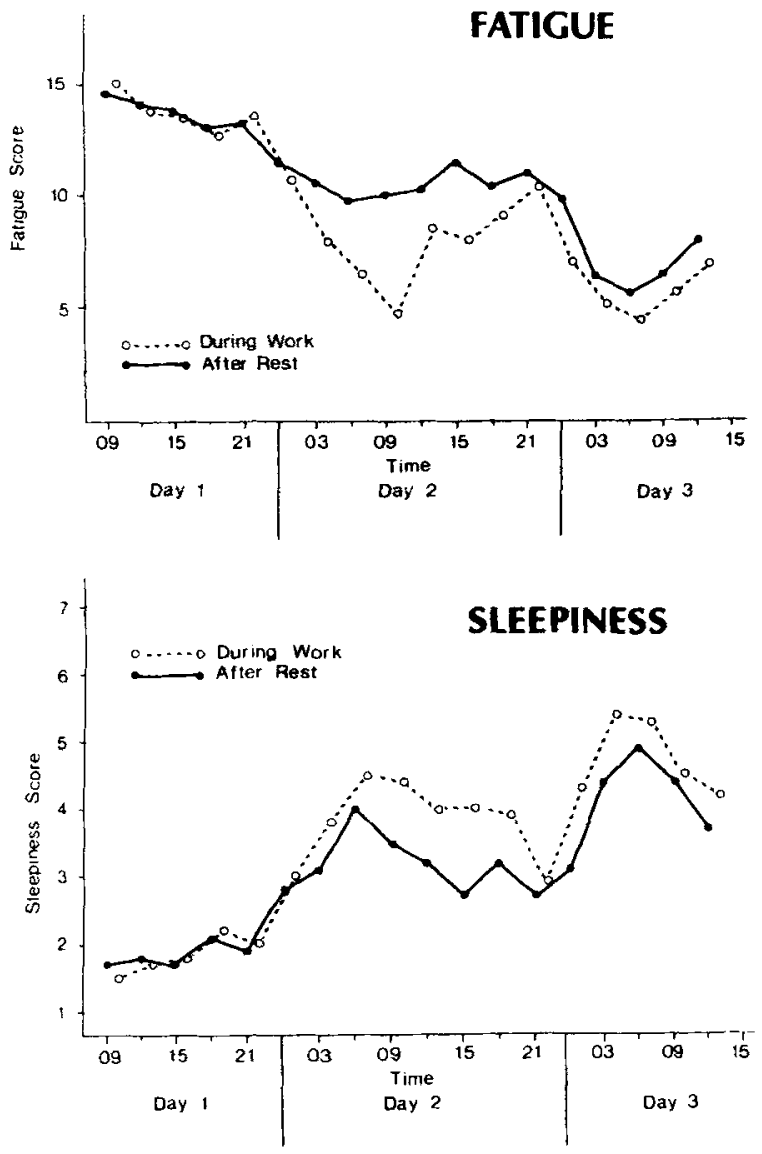

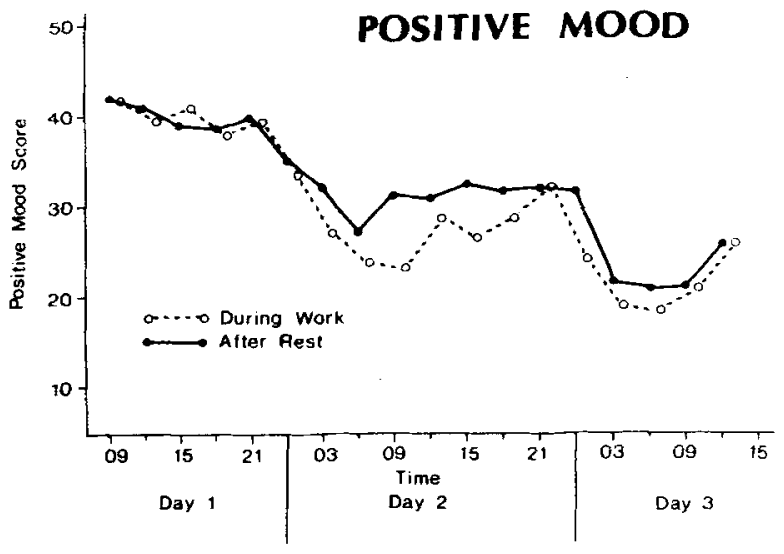

NEGATIVE MOOD

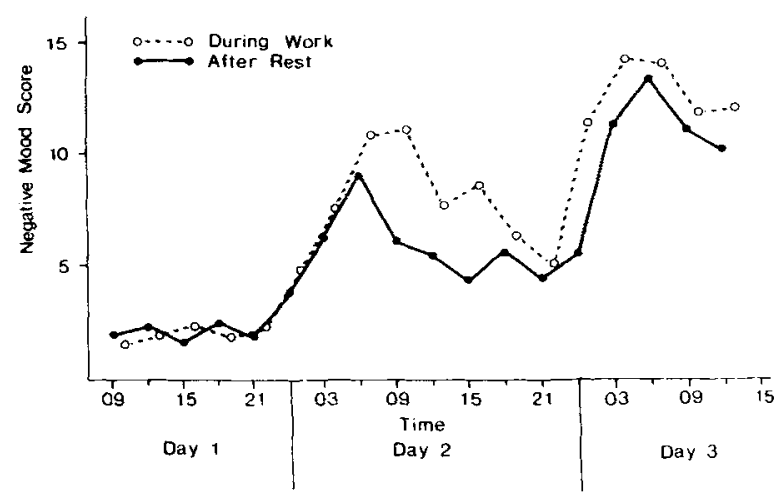

Figure 3. Self-report scales. The figure shows the differential changes in the subjective levels of fatigue, sleepiness, and positive and negative mood as a function of whether the subjects completed the scales after a short rest (solid line) or during a work session (dotted line). Larger numbers on the ordinate represent less subjective fatigue, greater subjective sleepiness, a more positive subjective mood, and a more negative subjective mood.

these were self-paced tasks and subjects did not need to sacrifice accuracy for speed. The 30 -min vigilance task was divided into 5-min periods for the analysis and is reported in terms of percentage of correct detections, since accuracy was sacrificed in this signal detection task.

\section{Serial Reaction Task (Four-Choice)}

The upper left panel in Figure 4 shows that the significant changes in performance on this task over blocks $[F(8,40)=35.03, p<.001]$ can be attributed to the declining number of correct responses made per minute rather than to an increase in the number of errors, as indicated by the interaction between correct responses versus errors and blocks $[F(8,40)=37.55, p<.001]$. Errors, however, did show an increase at $0500 \mathrm{~h}$ on the second night. The topography of the correct responses shows the same plateau effects seen in the subjective data, that is, stable performance until a drop at $0500 \mathrm{~h}$ the first night followed by relatively stable performance until $0500 \mathrm{~h}$ the second night.

\section{Logical Reasoning}

The results from the logical reasoning task, shown in the lower left panel of Figure 4, are similar in pattern to those of the serial reaction time task in that the changes in performance over blocks $[F(8,40)=29.96, p<.001]$ can be totally attributed to the declining number of correct responses rather than to changes in the number of errors. This interpretation is supported by the interaction of correct responses versus errors with blocks $[F(8,40)$ $=21.33, \mathrm{p}<.001]$.

\section{Encode/Decode}

The upper right panel shows the data from the encoding/decoding task with the correct responses and errors differentiated for both the encoding and decoding problems. Again the significant changes in performance over blocks $[F(8,40)=35.70, p<.001]$ can be accounted for by declines in the number of correct responses in both the encode and decode tasks, as indicated by the interaction of accuracy with blocks $[F(8,40)=25.88, p$ 

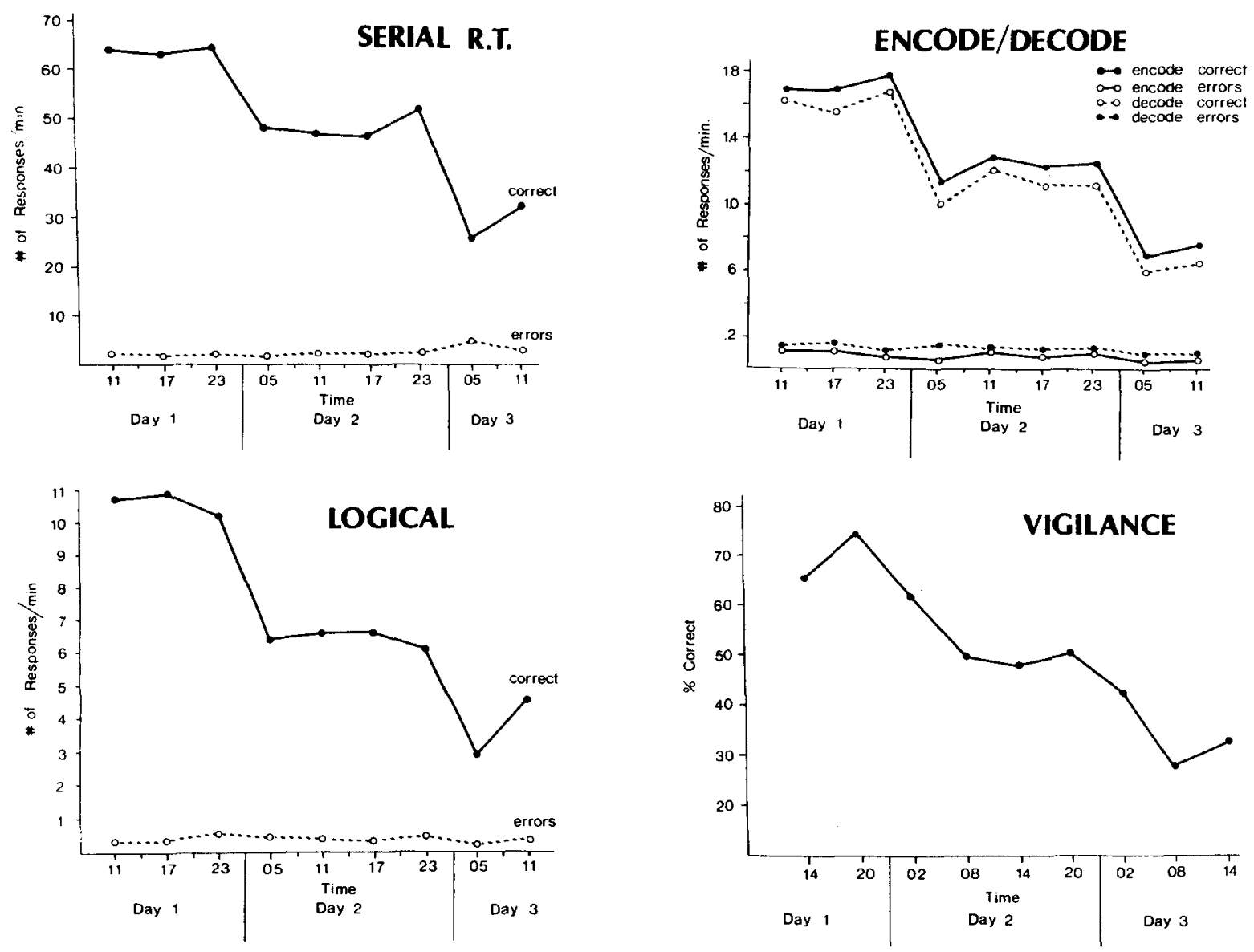

Figure 4. Performance tasks. The figure shows the changes in cognitive performance over the nine experimental blocks. For the serial reaction time and logical reasoning tasks (left panels), correct responses and errors are shown; for the encoding/decoding task (upper right panel), these correct responses and errors are further broken down by type of task. The vigilance task (lower right panel) is reported in terms of percentage of correct detections.

$<.001]$. The patterns of change are similar to those of the previous tasks, with the declining number of correct responses showing a plateau between significant drops in performance. In addition, the type of task (encode or decode) interacted with accuracy $[F(1,5)=55.74, p<$ $.001]$ in that subjects made more correct responses (closed circles connected by solid lines) and fewer errors (open circles connected by solid lines) on the encoding task than on the decoding task.

\section{Auditory Vigilance}

The 30-min auditory vigilance trials occurred about $3 \mathrm{~h}$ after the 10-min performance tasks. Since this task involved subjects making immediate time-dependent decisions concerning the occurrence of a signal, accuracy rates were affected, and therefore the data are reported in terms of the percentage of correctly detected signals. In addition, since the data in Blocks 3, 4, and 8 were lost for three subjects due to equipment failure, the data reported are for only 9 of the 12 subjects. Despite the reduced number of subjects, there was a significant decline in perfor- mance over blocks $[\mathrm{F}(8,64)=11.49, \mathrm{p}<.001]$, and the pattern was similar to the other tasks.

\section{Message Processing}

A primary objective of this experiment was to keep the subjects occupied continuously with a cognitive task that had some validity as a simulation of command and control tasks and that was engaging and progressive throughout the experiment. The message-processing task was used for this purpose. This task occurred within Sessions 1 and 3 of each 6-h block, and therefore data were available every $3 \mathrm{~h}$. Because many aspects of this task were subjective and the task was progressive, errors made by subjects could produce further errors before the experimenters could offer any correction. Therefore, estimates of processing time are reported independently of accuracy.

Although the messages were controlled neither for length nor for the number of questions asked per message, the work required was relatively homogeneous 
across the 18 message-processing sessions. A significant sessions effect $[F(17,85)=7.78, p<.001]$ emerged for the mean message-processing time (upper half of Figure 5). These data are similar to the data from the other tasks, except that the early sessions show an initial improvement in performance (reduced processing time) as the subjects learned how to respond to the various types of messages and questions. However, once the subjects became familiar with the task (by about $2100 \mathrm{~h}$ of the first day), the effect of the first night without sleep was to lower performance by increasing the length of time required to process the messages. This increase in processing time plateaued until about midnight of Day 2 and was followed by a dramatic decline in performance. The figure also shows some recovery at the end of the experiment.

In order to control for the number of questions in each message, the processing time for the questions themselves over the 18 sessions was analyzed independently of messages. These data are presented in the lower half of Figure 5. First consider the curve of open circles connected by broken lines, which represents an analysis of
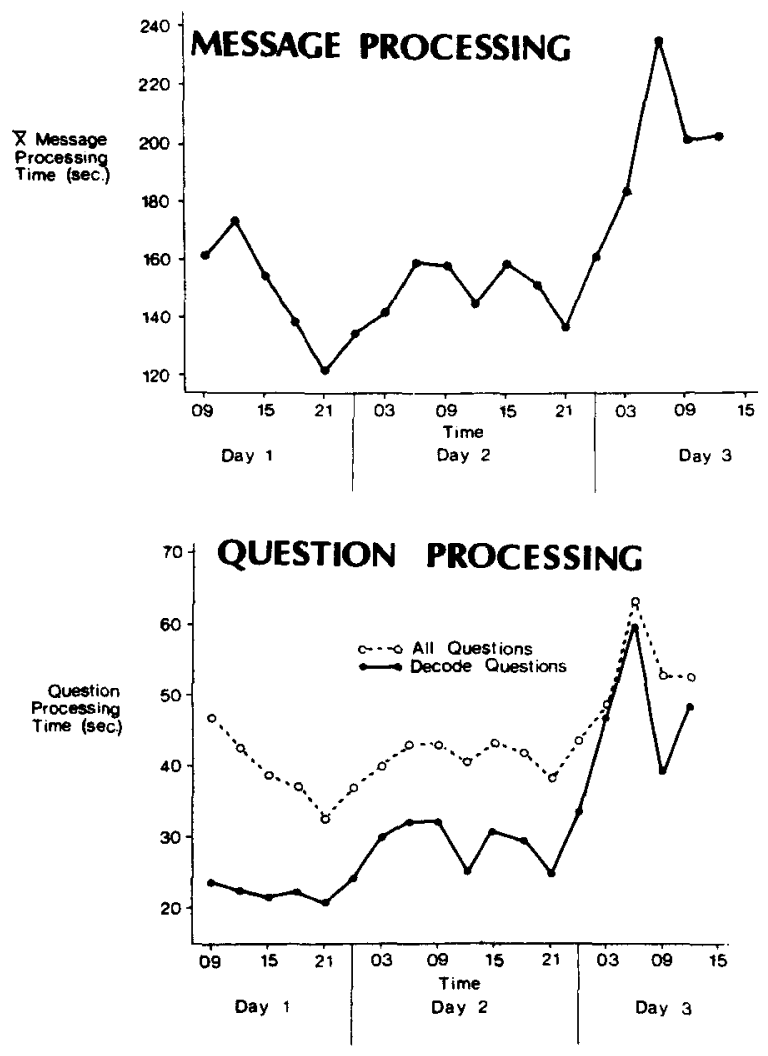

Figure 5. Message processing. The data in the upper panel represent the time taken by subjects to read, understand, and interpret messages, as well as to answer questions and file information over the 18 message-processing sessions. The lower panel represents the time required to answer the questions contained within the messages. The open circles show the mean question-processing time for all questions; the closed circles show the mean questionprocessing time for only the decoding questions. all questions asked in each session. There was a significant change over sessions $[F(17,85)=5.40, p<.001]$, showing essentially the same topography as the messageprocessing data. There appeared to be an initial learning period until about $2100 \mathrm{~h}$, followed by a decline that plateaued until about midnight of Day 2 , and then a dramatic decline in performance with some improvement near the end of the experiment.

To control for the content of the questions and to gain further precision, 12 specific questions were systematically included throughout the experiment as Priority 1 messages (see Method). These homogeneous questions asked subjects to decode the resource states of various units. The closed circles connected by solid lines in the lower half of Figure 5 depict these data. The significant change over sessions $[F(17,85)=13.37, p<.001]$ more closely resembled the data from the traditional cognitive tasks already reported. There was no learning effect since the task was already well-learned as part of the encode/decode task. In contrast to the data for all questions, the embedded decoding data more clearly show the lower performance (increased processing time) beginning from about $2100 \mathrm{~h}$ of the first night, the plateaued performance, and the dramatic decline in performance during the second night.

\section{DISCUSSION}

The basic tenet of this paper is that previous studies have provided conservative estimates of the performance degradation that might be expected as a function of sleep loss in sustained, intensive command and control operations. A comparison of the present results with previous findings supports this assertion.

For example, both Naitoh (1981) and Opstad et al. (1978) reported that, after a subject has gone one night without sleep, the number of correct responses on serial reaction tasks remains at about $90 \%$ of baseline. In the present study, reaction time performance fell to $76 \%$ of baseline over the same period. Naitoh (1981) reported that, during the second night, performance fell to $79 \%$ of baseline, whereas Opstad et al. (1978) reported that $90 \%$ performance levels were maintained for $90 \mathrm{~h}$ of sleep loss. In contrast, the present experiment showed that performance dropped to $43 \%$ of baseline during the second night.

For the logical reasoning task, Haslam (1982) reported performance levels of $71 \%$ and $35 \%$ of baseline following 24 and $48 \mathrm{~h}$ of sleep loss, respectively, whereas the present data showed drops to $57 \%$ and $26 \%$ during the same periods. For encoding/decoding performance, Haslam (1982) found no change until the second night, when performance was about $50 \%$ of baseline. In contrast, performance on this task in the present experiment declined to $72 \%$ of baseline during the first night and $41 \%$ the second night.

Data from the auditory task showed reductions to $66 \%$ and $38 \%$ of baseline at 24 and $48 \mathrm{~h}$, respectively. These 
data are comparable to those reported by Naitoh (1981), who found that correct auditory detections declined to $60 \%$ and $35 \%$ following 24 and $48 \mathrm{~h}$. However, the duration of the task in Naitoh's (1981) study was 50 min, whereas in this study the duration was only $30 \mathrm{~min}$. Since longer task durations result in greater performance decrements (Wilkinson, 1965), the present results are indicative of decrements in vigilance performance that are at least equivalent to or greater than those in Naitoh's (1981) study, once the differences in task duration are taken into account.

The fatigue, sleepiness, and mood scales all showed clear declines over the course of the experiment and showed plateaus similar to the performance tasks. To compare the present results with those from other studies (e.g., Naitoh, 1981), baseline was assumed to be $100 \%$ and reductions from baseline were assumed to indicate greater fatigue, less positive affect, more negative affect, and greater sleepiness. Naitoh's (1981) data showed that after $24 \mathrm{~h}$, subjects' self-reported states were $73 \%, 92 \%$, $48 \%$, and $87 \%$ of baseline, respectively, for the four scales. The reductions in this study (based on the 6-h means shown in Figure 2) were $43 \%, 70 \%, 29 \%$, and $64 \%$ of baseline after $24 \mathrm{~h}$ of wakefulness. After $48 \mathrm{~h}$, the present study continued to show greater degradations than Naitoh (1981) reported.

The subjective results also showed greater degradations than those reported by Mullaney et al. (1983), who repeated a 10 -min test battery every $10 \mathrm{~min}$ for $42 \mathrm{~h}$. For example, using normalized scores with baseline levels equalling $\mathrm{z}$ values of zero, Mullaney et al.(1983) showed that the greatest increase in subjective sleepiness occurred at $0500 \mathrm{~h}$ of the first night with a $\mathrm{z}$ value of about 2.3 , which subsequently recovered to a $\mathrm{z}$ value of about 1.5 by $1700 \mathrm{~h}$ of the following day. Using scores obtained from scales that occurred following short rest breaks, the present study showed similar increases in sleepiness at about $0500 \mathrm{~h}(\mathrm{z}=2.20)$ and $1700 \mathrm{~h}(\mathrm{z}=1.49)$. Recall, however, that the present self-report data showed greater increases in sleepiness on scales completed during work sessions. Thus, scores obtained from within work sessions showed a greater degree of subjective sleepiness around both $0500 \mathrm{~h}(\mathrm{z}=2.56)$ and $1700 \mathrm{~h}(\mathrm{z}=2.04)$ than those reported by Mullaney et al. (1983).

In general, the results reported here show that sustained cognitive activity resulted in greater mood and performance degradations than had previously been observed in experiments over similar time periods using similar dependent measures. Large decrements occurred during the first night of sleep loss (reductions of about $30 \%$ ), with performance becoming generally unacceptable during the second night (about 60\% reductions). Although the greatest declines coincided with the "trough" of the circadian cycle, the commonly reported increase in performance that accompanies the ascending limb of the circadian cycle was minimal. One reason for this might be that the interaction of sleep loss with the relatively demanding mental workload reduced, or depleted, the reserve ca- pacity normally available to subjects to increase performance during daytime periods. Although the performance results may also have been i. fluenced by analysis and procedural limitations (e.g., the self-report data were pooled across 6-h blocks, and the 10-min performance data were collected only every $6 \mathrm{~h}$ ), similar trends were evident in the hourly self-report data and in the messageprocessing task data which were averaged over 3-h intervals. Since the performance tasks reported were all from subject-paced tasks, errors showed marginal changes as a function of sleep loss, and the changes in performance were primarily due to reductions in the numbers of correct responses. It may be that work- or experimenterpaced tasks may yield a different pattern of results.

Finally, this study was intended to examine representative tasks and abilities in a simulated command and control setting. The relatively continuous nature of the task was designed to reduce monotony, and the tasks were embedded to make them less obtrusive, thereby reducing the influence of motivational changes on performance. In this regard, the message-processing-time and questionprocessing-time data provided estimates of performance degradation similar to those of the 10-min laboratory-type tasks. In addition, the importance of embedding tasks was demonstrated by the self-report results, with scales administered within work sessions showing differentially greater effects than scales administered following short rests. Similar results were observed for the embedded decoding task. These findings suggest that subjects are less able to maintain performance on tasks that are integrated within their primary function than on tasks that are distinct from their primary function. Future work will examine the differential effects of determining performance degradation by using discrete performance tasks versus embedding such measures within a relatively continuous task. Embedding tasks in this way should contribute to the development of a more realistic command and control simulation for the study of performance limits during sustained operations conditions.

\section{REFERENCES}

AINSwORTH, L. L., \& BishoP, H. P. (1971). The effect of a 48-hour period of sustained field activity on tank crew performance (HumRRO Tech. Rep. No. 71-16). Alexander, VA: Human Resources Research Office.

Alluisi, E. A. (1969). Sustained performance. In E. A. Bilodeau \& I. M. Bilodeau (Eds.), Principles of skill acquisition (pp. 59-101). New York: Academic Press.

BADDELEY, A. W. (1968). A 3 min reasoning test based on grammatical transformation. Psychonomic Science, 10, 341-342.

Banderet, L. E., Stokes, J. W., Francesconi, R., Kowal, D. M., \& NaIToH, P. (1981). Artillery teams in simulated sustained combat: Performance and other measures. In L. C. Johnson, D. J. Tepas, W. P. Colquhoun, \& M. J. Colligan (Eds.), Biological rhythms, sleep and shift work (pp. 459-477). New York: Spectrum.

Banks, J. H., Sternberg, J. J., Farrell, J. P., Debow, C. H., \& DELHAMER, W. A. (1970). Effects of continuous military operations on selected military tasks (BESRL Techn. Rep. No. 1166). Arlington, VA: U.S. Army Behavioral and Systems Research Laboratory. Colquhoun, W. P., Blake, M. J. F., E Edwards, R. S. (1968). Ex- 
perimental studies of shiftwork. I: A comparison of 'rotating' and 'stabilized' 4-hour shift systems. Ergonomics, 11, 437-453.

COOK, M. R., COHEN, H., ORNE, M. T. (1972). Recovery from fatigue (Tech. Rep. No. 55). Fort Detrick, MD: U.S. Army Medical Research and Development Command.

Drucker, E. H., CANON, L. D., \& WARE, J. R. (1969). The effects of sleep deprivation on performance over a 48-hour period (HumRRO Tech. Rep. No. 69-8). Alexander, VA: Human Resources Research Office.

HAGGARD, D. F. (1970). HumRRO studies in continuous operations (HumRRO Professional Paper No. 7-70). Alexander, VA: Human Resources Research Office.

HaRris, W., \& O'Hanlon, J. F. (1972). A study of recovery functions in man (Tech. Memorandum No. 10-72). Aberdeen Proving Ground, MD: U.S. Army Human Engineering Laboratory.

Harris, D. A., Pegram, G. V., \& Hartman, B. O. (1971). Performance and fatigue in experimental double-crew transport missions. Aviation. Space and Environmental Medicine, 24, 980-986.

HaSLAM, D. R. (1981). The military performance of soldiers in continuous operations: Exercise Early Call I and II. In L. C. Johnson, W. P. Tepas, W. P. Colquhoun, \& M. J. Colligan (Eds.), Biological rhythms, sleep and shift work (pp. 435-458). New York: Spectrum.

HASLAM, D. R. (1982). Sleep loss, recovery sleep and military performance. Ergonomics, 25, 163-178.

Heslegrave, R. J., \& FuREDY, J. J. (1979). Sensitivities of HR and T-wave amplitude for detecting cognitive and anticipatory stress. Physiology \& Behavior, 22, 17-23.

Hockey, G. R., \& Colquhoun, W. P. (1972). Diurnal variations in human performance: A review. In W. P. Colquhoun (Ed), Aspects of human efficiency (pp. 1-23). London: English Universities Press.

Hoddes, E., Zarcone, V., Smythe, H., Phillups, R., \& Dement, W. C. (1973). Quantification of sleepiness: A new approach. Psychophysiology, 10, 431-436.

JoHNSON, L. C. (1979). Sleep disturbances and performance. In A. N. Nicholson (Ed.), Sleep, wakefulness and circadian rhythm (NATO AGARD Lecture Series No. 105, pp. 8:1-8:17). Paris: NATO Advisory Group for Aerospace Research and Development.

JOHNSON, L. C. (1982). Sleep deprivation and performance. In W. B. Webb (Ed.), Biological rhythms, sleep, and performance (pp. 111 141). Chichester, England: Wiley.

Johnson, L. C., \& NAITOH, P. (1974). The operational consequences of sleep deprivation and sleep deficit (NATO AGARDograph No. 193). Paris: NATO Advisory Group for Aerospace Research and Development.

KJeLLBerg, A. (1977a). Sleep deprivation and some aspects of performance. I: Problems of arousal changes. Waking and Sleeping, 1, 139-143.

KJELLBERG, A. (1977b). Sleep deprivation and some aspects of performance. II: Lapses and other attentional effects. Waking and Sleeping, 1, 145-148.

KJELLBERG, A. (1977c). Sleep deprivation and some aspects of perfor- mance. III: Motivation, comment and conclusions. Waking and Sleeping, 1, 149-153.

Morgan, B. B., Brown, B. R., \& Alluisi, E. A. (1974). Effects of sustained performance of 48 hours of continuous work and sleep loss. Human Factors, 16, 406-414.

Mullaney, D. J., Kripke, D. F., Fleck, P. A., \& Johnson, L. C. (1983). Sleep loss and nap effects on sustained continuous performance. Psychophysiology, 20, 643-651.

NaITOH, P. (1976). Sleep deprivation in human subjects: A reappraisal. Waking and Sleeping, 1, 53-60.

NaItoH, P. (1981). Circadian cycles and restorative power of naps. In L. C. Johnson, D. 1. Tepas, W. P. Colquhoun, \& M. J. Colligan (Eds.), Biological rhythms, sleep and shift work (pp. 553-580). New York: Spectrum.

NaITOH, P., ENGLUND, C. E., \& RYMaN, D. (1982). Restorative power of naps in designing continuous work schedules (NHRC Tech. Rep. No. 82-25). San Diego: Naval Health Research Center.

NaItoh, P., \& TownSEND, R. E. (1970). The role of sleep deprivation in human factors. Human Factors, 12, 575-585.

Opstad, P. K., Ekanger, M., Nummestrad, M., \& RaAbe, N. (1978). Performance, mood and clinical symptoms in men exposed to prolonged, severe physical work and sleep deprivation. Aviation, Space and Environmental Medicine, 49, 1065-1073.

ShePhard, R. J. (1982). Physiology and biochemistry of exercise. New York: Praeger.

Wilkinson, R. T. (1964). Effects of up to 60 hours of sleep deprivation on different types of work. Ergonomics, 17, 175-186.

WiLkinson, R. T. (1965). Sleep deprivation. In O. G. Edholm \& A. Bacharach (Eds.), The physiology of human survival (pp. 399-430). New York: Academic Press.

WiLkinson, R. T. (1969a). Sleep deprivation: Performance tests for partial and selected sleep deprivation. Progress in Clinical Psychology, 8, 28-43

WILKINSON, R. T. (1969b). Some factors influencing the effect of environmental stresses upon performance. Psychological Bulletin, 72, 260-272.

Wilkinson, R. T., Edwards, R. S., \& Haines, E. (1966). Performance following a night of reduced sleep. Psychonomic Science, 5, 471-472.

Wilkinson, R. T., \& Houghton, D. (1975). Portable four-choice reaction time test with magnetic tape memory. Behavior Research Methods \& Instrumentation, 7, 441-446.

\section{NOTE}

1. Although the results of these studies are directed toward a military command and control environment, the results of this research program are also applicable to a variety of crisis situations requiring sustained, intensive, cognitive and vigilant activity (e.g., search and rescue, fire fighting, medical and civil emergencies), as well as to noncrisis situations involving alternative work cycles (e.g., shift-work and extended-duty requirements). 